Cahiers du MONDE RUSSE

\section{Cahiers du monde russe}

Russie - Empire russe - Union soviétique et États indépendants

$57 / 2-3 \mid 2016$

Famille et mobilité sociale en Russie, XVIe-XVIIle siècles

\title{
Rapatriement, genre et mobilité sociale
}

La liste des captifs rapatriés de Crimée par Timofej Hotunskij (1649) Repatriation, gender and social mobility: The register of captives returned from Crimea with Timofei Khotunskii (1649)

\section{Aleksandr Lavrov}

\section{OpenEdition}

\section{Journals}

\section{Édition électronique}

URL : http://journals.openedition.org/monderusse/8379

DOI : $10.4000 /$ monderusse. 8379

ISSN : $1777-5388$

\section{Éditeur}

Éditions de l'EHESS

\section{Édition imprimée}

Date de publication : 1 avril 2016

Pagination : 667-685

ISBN : 978-2-7132-2541-3

ISSN : $1252-6576$

\section{Référence électronique}

Aleksandr Lavrov, «Rapatriement, genre et mobilité sociale », Cahiers du monde russe [En ligne], 57/2-3 | 2016, mis en ligne le 01 avril 2019, Consulté le 21 avril 2019. URL : http:// journals.openedition.org/monderusse/8379; DOI : 10.4000/monderusse.8379

Ce document a été généré automatiquement le 21 avril 2019

(c) École des hautes études en sciences sociales 


\title{
Rapatriement, genre et mobilité sociale
}

\author{
La liste des captifs rapatriés de Crimée par Timofej Hotunskij (1649) \\ Repatriation, gender and social mobility: The register of captives returned from \\ Crimea with Timofei Khotunskii (1649)
}

\section{Aleksandr Lavrov}

1 Au printemps 1649, l'envoyé du tsar au Khanat de Crimée Timofej Hotunskij rentra en Russie avec une caravane de prisonniers rachetés ${ }^{1}$. La liste des anciens prisonniers, quoiqu'incomplète, comptait 898 personnes. Un pourcentage particulièrement élevé de femmes et d'enfants dans cette caravane apparaît comme un trait inhabituel. Il s'explique sans aucun doute par le statut diplomatique de Hotunskij, qui lui permettait de bénéficier d'une escorte tatare jusqu'à la frontière moscovite. Dans ces conditions, les femmes et enfants libérés pouvaient se sentir dans une relative sécurité pendant leur passage par les steppes. Citons la biographie laconique, assez typique, d'une captive rapatriée, celle d'Ul 'jana Malyhina, notée contre son nom dans la liste : « ...] fille du cadet de province (syn bojarskij) Trofim Malyhin de Kursk, passa 25 ans en captivité ». Ul'jana sortit de Crimée avec deux fillettes adoptées : l'une, Natal'ja, âgée de cinq ans, se souvenait encore qu'elle était originaire du village de Kobylina, tandis que l'autre, âgée de deux ans et capturée "ce printemps ", ne savait ni son propre prénom, ni le prénom de son père ${ }^{2}$. Les fillettes furent-elles adoptées par Ul'jana Malyhina au moment de départ de la caravane pour la Moscovie, afin de les protéger au cours d'un voyage difficile et dangereux, ou bien cette famille recomposée s'était-elle constituée en captivité ? Il est évident en tout cas que la femme, célibataire, n'aurait pu subvenir seule aux besoins de ces deux enfants et que les autorités moscovites devaient prévoir des mesures pour les soutenir, ce qui fut fait. D'autres cas exigeaient également l'attention particulière des autorités. Par exemple, celui d'une captive qui resta complètement anonyme : « Jeune fille captive, mineure, ne se souvient pas de son nom, ni de celui de son père, capturée cette année $»^{3}$. Dans la liste de Hotunskij, 119 personnes étaient incapables de citer leurs origines, certaines affirmaient ne se souvenir ni de leur nom, ni de leur prénom. Cela causait des difficultés aux sous-secrétaires (pod'jačie) qui devaient rédiger la liste des captifs. Non seulement il 
fallait donner à ces captifs des noms et des prénoms, mais encore était-on obligé de les munir d'une identité sociale et d'une appartenance locale, car en Moscovie un statut comportait obligatoirement une indication géographique (on ne pouvait être, par exemple, une posadskaja (taillable des villes) tout court, mais seulement une posadskaja-de-telle-ville).

2 Certaines indications biographiques dans la liste suscitent des questions. Par exemple, « la fille captive âgée de 15 ans, affirme qu'elle est issue de parents russes, son prénom est Kutlukiz » ${ }^{4}$. Évidemment, Kutlu-Kyz, qui signifie en tatare " une fille heureuse », reçut son prénom en captivité, et elle ne pouvait (ou ne voulait) rien raconter aux autorités sur elle-même. Cependant, les sous-secrétaires moscovites ne voyaient apparemment aucun inconvénient à une telle déclaration identitaire. De retour en Moscovie, Kutlu-Kyz fut sans aucun doute intégrée dans la communauté moscovite 5 . L'étude de la liste ne permet pas de supposer qu'un quelconque rapatrié eût été rejeté ou renvoyé. Nous reviendrons sur les fondements de cette politique de "porte ouverte" qui ne demandait aux nouveaux arrivants rien d'autre que la volonté de rejoindre la Moscovie.

3 Avant d'exposer le problème étudié, il faut dire quelques mots sur la place de notre sujet dans l'étude de la société moscovite et de ses structures de parenté. Certes, les anciens captifs et captives, revenus en Moscovie, présentent une minorité minuscule en comparaison avec d'autres groupes sociaux. Mais, parmi les autres, ce groupe était toujours bien repéré par les statistiques officielles et c'est à l'égard de ce groupe que les autorités moscovites appliquaient toutes les techniques du contrôle qui leur étaient accessibles. Ensuite, ce groupe posait aux autorités des problèmes inédits, car ses représentants, demandant une assistance sociale, avaient aussi des problèmes d'identité, dus à leur réintégration.

4 Il faut ajouter quelques précisions sur les données de la liste et sur l'organisation de la banque de données qui a permis leur formalisation. Les sous-secrétaires moscovites ont classé tous les captifs et captives dans deux registres. Dans le premier, on trouve, inscrits dans un ordre qui ne correspond ni à l'alphabet, ni à la provenance géographique, les adultes, et les mineurs qui sortaient de captivité sans parents. Pour chacun d'eux sont indiqués le nom, le prénom, le village ou la ville, le district d'origine, la durée de la captivité et le lieu de capture (il n'était pas toujours nécessaire de préciser le sexe, dénoté par la forme des prénoms russes). Les données sur l'âge et le statut marital chez les femmes sont assez incertaines. S'il est indiqué qu'une captive passa vingt ans en Crimée, cela permet au moins de supposer qu'il s'agit d'une femme adulte. Il semble que termes « fille » (devka) et « femme » (žonka) renvoient simultanément à l'âge et au statut marital et ne peuvent être traduits que comme synonymes de « femme adulte».

Dans le second registre sont inscrits les enfants qui sortaient de captivité avec leurs parents ou leurs parents adoptifs. On indiquait normalement le nombre de mineurs, leur sexe et leur âge, mais pas nécessairement leur prénom (les causes de cette omission seront discutées plus loin). Le cas d'Ul'jana Malyhina, citée plus haut, est un bon exemple. Ainsi, les mineurs qui sortaient avec leurs parents n'avaient pas droit à une entrée séparée dans la liste, mais étaient introduits après le nom de leur mère. En comparant l'âge des enfants et la durée de la captivité de leur mère, on peut parfois établir s'il s'agit d'enfants capturés avec leurs mères, qui sont absolument minoritaires, ou bien d'enfants nés en captivité, majoritaires. 
Compte tenu des particularités de la source, j'ai décidé de ne retenir pour cette étude que les données du premier registre, c'est-à-dire les adultes et les mineurs qui sortaient de captivité sans parents, soit 723 personnes. Deux raisons ont été décisives dans cette approche. Premièrement, les données sur les enfants sortis avec leurs parents sont tellement pauvres et asystématiques que leur étude ne donne pas de résultats. Dans quelques cas, même le sexe des enfants n'est pas mentionné. Deuxièmement, pour les enfants nés en captivité, deux éléments constitutifs sont absents: la capture, car eux-mêmes ne furent jamais capturés, et l'origine géographique, pour laquelle on pourrait indiquer « de Crimée ».

Tableau 1. Pourcentage des hommes et des femmes

\begin{tabular}{|l|l|l|}
\hline & Adultes et mineur(e)s sans parents & Mineur(e)s \\
\hline Captives & 554 & 83 \\
\hline Captifs & 169 & 84 \\
\hline Sans indication de sexe & 0 & 8 \\
\hline Total & 723 & 175 \\
\hline
\end{tabular}

$7 \quad$ Le haut pourcentage de femmes (554 contre 169 hommes) ${ }^{6}$ est intéressant de deux points de vue. Premièrement, les femmes captives sont intéressantes comme telles. Dans les années 1990, Nancy Shields Kollmann, réfutant certaines légendes historiographiques, a montré que le statut légal des femmes en Moscovie n'était pas aussi subordonné qu'on le croyait ${ }^{7}$. En allant dans la même direction, Nada Boškovska a montré comment ce statut fut réalisé en pratique. L'image dressée par l'historienne est très contradictoire: les femmes moscovites jouaient des rôles importants, mais en même temps, elles étaient menacées par la violence dans la vie quotidienne ${ }^{8}$. Pourtant, la guerre était un facteur fondamental de la vie moscovite, et ce n'est pas par hasard que Sergej M. Solov'ev définit la société moscovite comme "une société de guerre, une société de service (boevoe, služiloe) ». Un aperçu de la législation suffit à montrer que les captives furent en réalité exclues de la hiérarchie des rançons et, par conséquence, exclues des rachats financés par l'État, reléguées au rançonnement familial' ${ }^{9}$. Il serait intéressant de comprendre comment ce statut légal influençait la situation réelle des femmes pendant les hostilités.

Deuxièmement, tandis que les captifs étaient constitués au moins partiellement d'hommes d'armes, capturés sur le champ de bataille, les captives étaient des non-combattantes par définition. Ainsi, l'étude de l'origine géographique des captives et de la durée de leur captivité permet de mieux apprécier les privations de la population civile de la Moscovie pendant les incursions tatares.

\section{L'origine géographique et la pyramide démographique}

Pour la majorité absolue des captives (479 sur 554), la ville ou le district de leur origine sont indiqués. Les captives viennent d'un large secteur du territoire moscovite, qui commence par le district de Smolensk à l'ouest et finit par Alatyr' sur le fleuve Sura à l'est 
(dans la Tchouvachie actuelle). Pour mieux interpréter les données sur la provenance des captives, un classement des villes et districts est nécessaire, qui pourrait être différent de la division officielle moscovite. J'ai choisi comme critère le degré de protection du lieu contre les invasions tatares. Selon toute logique, les villes et districts situés sur la ligne fortifiée de Belgorod (Belgorodskaja čerta), dont la construction, commencée en 1635, n'était pas encore achevée en 1649, devraient être les plus exposés. Il s'agit de Sokol'sk, Voronež, Userd' et Valujki. ${ }^{10}$

Dans le deuxième groupe, j'ai réuni les villes situées entre la ligne Belgorod et l'ancienne ligne d'abattis (Zasečnaja čerta). Dans ce groupe plutôt hétérogène, j'ai réuni les « villes de la steppe » (polevye goroda), les " villes frontières" (ukrainnye goroda) et les « villes de la Sévérie » (severskie goroda). Selon la terminologie moscovite des années 1620, le premier terme désignait les bourgades fortifiées destinées à bloquer les offensives tatares (Elec, Lebedjan', Livny, Kursk, Donkov et Oskol) ${ }^{11}$. Parmi les "villes frontières» on comptait Dedilov, Čern', Epifan', Orel, Kromy, Mihajlov, Pronsk, Mcensk, Gremjačev, Ryl 'sk, Pečerniki et le canton de Komarickaja. Selon toute apparence, les populations de ces villes devaient être moins exposées au risque de capture que celles de la ligne Belgorod.

Le troisième groupe est formé par les villes situées sur l'ancienne ligne. Si on la suit de l'ouest vers l'sst, ce sont Kozel'sk, Odoev, Krapivna, Tula, Venev, Zarajsk, Perejaslavl '-Rjazanskij, Rjažsk, Rjazan' et Šack. L'ancienne ligne présentait une chaîne d'abattis et de fortifications qui pouvaient arrêter les attaquants pour quelques heures mais, en l'absence de commandement militaire central capable d'envoyer des renforts, le temps gagné ne donnait pas d'avantages immédiats, ce qui devint évident surtout pendant le Temps des Troubles ${ }^{12}$.

Le quatrième groupe est formé par les villes situées le long de la basse Oka (Kaluga, Aleksin, Serpuxov, Kašira et Kolomna). Quand les Tatares dépassaient l'ancienne ligne, ils arrivaient sur la rive de l'Oka qui formait la dernière barrière naturelle avant Moscou. Au $\mathrm{XVI}^{\mathrm{e}}$ siècle encore, les forces principales moscovites étaient déployées sur l'oka où elles attendaient les Tatares. Une tentative de traverser l'oka offrait une des meilleures possibilités pour une bataille générale.

Le cinquième groupe est formé par les villes qui se trouvaient sur la haute Oka et autour de Brjansk (Brjansk, Bolhov, Karačev, Novosil', Meščevsk, Belev, Peremyšl', Vorotynsk, Lihvin et Trubčevsk). Dans son étude classique sur la Moscovie au Xvi ${ }^{\mathrm{e}}$ siècle, Mixail N. Tihomirov propose pour ces villes une notion spéciale : «les villes du pays de Brjansk et les villes situées au-delà de l'oka (zaocko-brjanskij kraj) $\aleph^{13}$. Il existe une raison sérieuse pour classer ces villes dans un groupe à part. Leur protection pouvait être double (par l'oka et par l'ancienne ligne), ou simple (par l'oka), mais il faut y ajouter les forêts qui affaiblissaient la menace tatare. Il y a encore une cause particulièrement intéressante: ici, sur la haute Oka, même les petites bourgades étaient dotées de fortifications importantes, comme dans le cas de Meščovsk. Ces fortifications, construites souvent non loin les unes des autres, amélioraient considérablement la protection de la population. L'existence de ces bourgades est due à la longue persistance de petites principautés sur la haute $\mathrm{Oka}$; et même après la disparition de ces dernières, les bourgades restèrent comme leurs vestiges ${ }^{14}$. Il faut noter que ce type de fortifications, un réseau de forteresses prêtant l'abri à la population locale, ressemblait au système ukrainien de protection des populations frontalières. 
Le sixième groupe est formé par les villes autour et derrière Moscou et Smolensk. Le septième, enfin (représenté dans la liste par une seule captive, provenant du Grand-duché de Lituanie), réunit les villes étrangères. Les résultats de la systématisation des données de la liste peuvent être présentés de manière suivante :

Tableau 2. Provenance géographique

\begin{tabular}{|l|l|l|}
\hline & Nombre & $\%$ \\
\hline Les villes sur la ligne Belgorod & 7 & 1 \\
\hline Les villes entre la ligne Belgorod et l'ancienne ligne & 187 & 34 \\
\hline Les villes sur l'ancienne ligne d'abattis & 112 & 20 \\
\hline Les villes du pays de Brjansk & 96 & 17 \\
\hline Les villes sur l'oka & 47 & 8,9 \\
\hline Les villes derrière l'Oka & 21 & 4 \\
\hline Étrangères & 1 & 0,1 \\
\hline Inconnue & 83 & 15 \\
\hline Total & 554 & 100 \\
\hline
\end{tabular}

15 Contre toute attente, les résidentes des villes de la future ligne Belgorod semblent épargnées (seulement 7 capturées, $1 \%$ ). Ces villes, suffisamment fortifiées et peuplées majoritairement par des mousquetaires (strel'cy) et les cosaques de service n'attiraient pas les Tatares. Un bon exemple en est la ville de Valujki qui, pendant les années 1620, n'avait pas de paysans, ni de cadets de province, et son district pas davantage : toute la population, formée de gens de service, vivait « sous la protection des fortifications » (une captive en est originaire $)^{15}$. Plus intéressant encore semble le fait que la ville de Kozlov, fondée en 1635, qui se développa très vite comme un centre très important, ne fournit aucune captive à la liste de Hotunskij ${ }^{16}$. Tant que la ligne était encore perceptible, les Tatares, évidemment, préféraient la passer le plus vite possible pour prendre des captifs dans les régions moins protégées.

En prenant en compte tout cela, les résidentes des villes et des districts entre la ligne Belgorod et l'ancienne ligne, c'est-à-dire celles du deuxième groupe, s'avèrent avoir été les plus exposées. Elles donnent 187 captives, soit $34 \%$ du total, dont l'origine locale est connue. Si on élimine du calcul les captives dont l'origine locale est inconnue, on pourrait dire qu'elles forment la majorité de celles dont l'origine locale est connue. La population ici était tout simplement livrée aux invasions tatares.

17 Le troisième et le quatrième groupe, les villes de l'ancienne ligne et celles de la région de Brjansk, semblent avoir un peu moins souffert des invasions tatares. Elles donnent respectivement 112 et 96 captives ( $20 \%$ et $17 \%$ ). Bien sûr, on pourrait noter que certaines villes de ces groupes étaient épargnées par les attaques tatares à partir du Temps des Troubles. Mais dans ces deux groupes, on avait des villes et des districts touchés tout 
récemment. On ne peut être étonné par le cas particulier de Tula, «qui représentait le point où se croisaient tous les chemins utilisés par les Tatares » et qui fut par conséquent souvent touchée ${ }^{17}$.

Le cinquième groupe, les villes sur l'Oka, qui donnent 47 captives $(8,9 \%)$, semble un peu plus protégé. Mais quelques districts de ce groupe furent encore touchés pendant l'incursion tatare au printemps 1649.

Le seul groupe qui fut presque épargné est formé par les districts situés au-delà de l'oka (Moscou, Smolensk, etc.) avec 21 captifs (4\%). Tandis que chaque ville, à l'exception de Moscou, est représentée par une ou deux captives, on pourrait penser qu'il s'agit de voyageuses qui furent capturées loin de leur lieu de résidence.

Pour mieux comprendre la pertinence de ces données, il faut revenir aux représentations de l'efficacité du système moscovite de fortifications dans l'historiographie. Selon la vision traditionnelle, formulée par A.A. Novosel'skij, les lignes fortifiées moscovites étaient efficaces et présentaient des avantages incontestables sur le système de défense polonais ${ }^{18}$. Des doutes furent exprimés par V.P. Zagorovskij, qui écrit qu' "avant la construction de la ligne Belgorod, les mérites du "système défensif moscovite" étaient assez douteux et visibles seulement si on les compare avec l'absence d'un système semblable en Pologne $»^{19}$. Un jugement beaucoup plus nuancé fut proposé par Gennadij A. Sanin qui remarque que dans le cas où la ligne était percée, la population civile ne trouvait plus de refuge, tandis que le système polonais, fondé sur les petites forteresses, avait ses avantages ${ }^{20}$.

21 Les données de la liste de Hotunskij ne permettent pas de prendre position dans cette discussion, car au moment de sa composition, la ligne fortifiée de Belgorod venait d'être achevée. La majorité des captives étant tombée en captivité avant la fin des travaux, il est impossible d'utiliser les données de la liste pour juger de l'efficacité de la ligne Belgorod. On peut par ailleurs prendre en considération que certaines captives, présentes dans la liste, tombèrent en captivité pendant le Temps des Troubles, quand l'ancienne ligne de fortifications ne fonctionnait plus.

22 Cependant, même un jugement très succinct ne peut être qu'assez pessimiste. On voit que même au milieu du XVII ${ }^{\mathrm{e}}$ siècle un grand secteur du territoire moscovite était exposé aux incursions tatares. Le fait que les Tatares de Crimée n'entreprenaient pas de grandes expéditions vers le centre de la Moscovie, mais se contentaient de petites incursions frontalières, pourrait s'expliquer non par l'efficacité de la défense moscovite, mais plutôt par le fait que la population autour de la ligne était tellement exposée aux incursions qu'il était plus simple d'y prendre des captifs et de les emmener en Crimée. De ce point de vue, la population autour de la ligne jouait le rôle d'un barrage humain.

23 Les données directes sur l'âge des anciennes captives sont absentes de la liste. Mais l'étude du temps qu'elles ont passé en captivité pourrait donner quelques données indirectes.

Tableau 3. Temps de captivité

\begin{tabular}{|l|l|l|l|l|l|l|l|l|}
\hline & 0,5 & $\begin{array}{l}1-9 \\
\text { années }\end{array}$ & $\begin{array}{l}10-19 \\
\text { années }\end{array}$ & $\begin{array}{l}20 \\
\text { années }\end{array}$ & $\begin{array}{l}21-29 \\
\text { années }\end{array}$ & $\begin{array}{l}30-40 \\
\text { années }\end{array}$ & Inconnu & Ensemble \\
\hline Nombre & 112 & 31 & 72 & 156 & 63 & 32 & 88 & 554 \\
\hline
\end{tabular}




\begin{tabular}{|l|l|l|l|l|l|l|l|l|}
\hline$\%$ & $20 \%$ & $6 \%$ & $13 \%$ & $28 \%$ & $11 \%$ & $6 \%$ & $16 \%$ & $100 \%$ \\
\hline & & & & & & & & \\
\hline
\end{tabular}
e n'ai trouvé qu'une famille non séparée dans la captivité, celle du paysan Ivan Terent'ev qui sortit de captivité avec sa femme Orina Ivanova ( $\left.{ }^{\circ} 88-89\right)$. Tous deux étaient originaires du district de Karačev, tous deux furent capturés «il y a 20 ans ${ }^{26}$. Ainsi, l'hypothèse s'impose que les époux restèrent ensemble pendant toute la captivité. Il est particulièrement intéressant qu'il s'agisse ici d'une famille paysanne, pour le rachat de laquelle les autorités moscovites ne voulaient jamais payer grand-chose, tandis que, dans la logique de la captivité de rançon, on devait mieux traiter les familles nobles ou 
marchandes, pour lesquelles on pouvait demander une rançon privée exorbitante. Cette famille ne fut pas séparée selon une autre logique, probablement pour affaiblir la volonté de fuir.

La présence dans la liste de Hotunskij de trois familles créées en captivité montre que ce phénomène pouvait aussi être toléré par les propriétaires tatares. Il est impossible d'imaginer que Stepan Dimitriev et sa femme aient été mariés avant la captivité : il était capturé «il y a 30 ans", et sa femme "il y a 20 ans »; elle venait de Perejaslavl '-Rjazanskij, tandis qu'il ne pouvait indiquer son ancien lieu de résidence. Ils avaient deux enfants, un fils de cinq ans et une fillette de deux ans, ce qui pourrait indiquer un mariage qui n'a pas duré plus d'une décennie. La deuxième famille inscrite dans la liste, Ivan Stepanov et Feodos'ja Romanova avec leurs deux filles, s'inscrit dans le même modèle, car le mari venait de Carev-Borisov, et la femme du district de Kaluga. Il est intéressant que l'entrée de la liste insiste sur la parenté de Stepanov, en notant que c'est lui qui «a conçu » des enfants avec sa femme ${ }^{27}$. Il est évident qu'une telle famille pouvait être tolérée ou même protégée, mais ce n'est pas la règle générale. Dans le cas de la troisième famille, la femme était probablement soumise à l'exploitation sexuelle. Marinka Jur'eva sortit de captivité avec son mari, le paysan Ivan Vasil'ev (district de Belev) et un fils de sept ans qu'elle "a conçu chez les Nogays (prižila v nagaeh) ${ }^{28}$. Vasil'ev et Jur'eva furent-ils capturés ensemble, ou se marièrent-ils en captivité ? Étaient-ils ensemble en captivité, ou Vasil'ev ne retrouva-t-il sa femme qu'à la veille de sa libération, dans la caravane de Hotunskij? Toutes ces questions restent ouvertes.

En revanche, la liste contient un grand nombre de femmes (135, ou $24 \%$ ), qui revenaient de captivité avec des enfants. Il faut dire que ste de Hotunskij permet pour la première fois de mesurer le poids du phénomène d'exploitation sexuelle, qui est presque tabou dans les récits des anciennes captives. Très souvent (dans 84 cas) on trouve la remarque " conçu chez les Tatares " ou " conçu chez les Nogays » (" prižit v tatareh », "prižit v nagaeh»). Bien sûr, il ne s'agit que d'une estimation. Il va de soi que toute captive soumise à l'exploitation sexuelle n'avait pas nécessairement des enfants, et que les captives qui eurent des enfants avec ceux qui profitaient de leur dépendance n'avaient pas forcément toutes réussi à les garder auprès d'elles et à les emmener en Moscovie.

En même temps, outre leurs enfants, les captives emmenaient aussi en Moscovie des enfants qui avaient été capturés avec elles, des enfants adoptés en captivité, des enfants nés en captivité d'un concubinage avec un autre captif moscovite ou tout simplement des enfants sur l'origine desquels la liste préférait ne rien dire.

Il n'est pas simple de séparer le premier groupe, ceux qui furent capturés avec leurs mères. Normalement, aucune remarque spéciale ne concerne ces enfants, mais, quand l'âge de l'enfant est indiqué et qu'il dépasse clairement la durée de la captivité de la mère, une telle identification s'impose. Cependant, un tel calcul nécessite qu'on sache l'âge de l'enfant et la durée de la captivité de la mère. Finalement, j'ai identifié onze enfants ayant été capturés avec leurs mères. Le deuxième groupe est identifiable grâce à la remarque particulière « conçu avec un homme russe » (prižila s ruskim čelovekom); il ne compte que quatre enfants. Le troisième groupe, six priemyši, est formé par des enfants qui semblent avoir été séparés de leurs parents pendant la capture et adoptés par des captifs adultes, ce qui était indispensable pour leur survie et leur rapatriement. L'origine d'onze autres enfants reste inconnue. 
33 En analysant cette liste, il est toujours important de retourner vers le groupe le plus nombreux, c'est-à-dire vers les enfants des esclaves russes et de leurs propriétaires tatares et nogays. Il est intéressant que ces enfants n'avaient pas été retenus par les autorités du Khanat, bien que leurs pères aient pu être musulmans.

Ce dernier fait, jamais mentionné ou apprécié dans l'historiographie, m'impressionna tellement pendant la première lecture de la liste, que j'ai formé l'hypothèse selon laquelle les garçons, qui pouvaient être intégrés dans la société criméenne, étaient obligés de rester, tandis que les filles pouvaient partir avec leurs mères. Dans ce dernier cas, le nombre des filles rapatriées devait clairement dépasser le nombre de garçons. En réalité, on constate l'opposé. 83 filles et 84 garçons vinrent avec leurs mères à Moscou, tandis que le sexe de huit enfants n'est pas indiqué, ce qui montre l'équité absolue de la part des autorités criméennes et permet d'écarter toute hypothèse d'un traitement différent des enfants selon le sexe $\mathrm{e}^{29}$.

L'attitude des autorités de Crimée se dessine ici clairement: à la condition du consentement des propriétaires, on accordait le même droit de départ aux captives et aux captifs. Ici, on pourrait voir une différence entre l'esclavage ottoman, qui était un élément important de l'intégration sociale, et l'esclavage criméen, qui était très important pour l'économie, l'agriculture et le prestige des propriétaires, mais qui ne développa pas la fonction d'intégration. En ce sens, la société criméenne garda sa spécificité de société nomade, descendante de la Horde d'Or, qui rendait l'intégration des «Autres» très difficile. Même affranchis et restés en Crimée, les anciens esclaves moscovites constituaient une «société parallèle ». L'option choisie par les autorités moscovites était à l'opposé.

\section{Le rapatriement comme un laboratoire identitaire}

L'étude de la distribution sociale des captives permet de découvrir un autre côté du phénomène de rapatriement.

Tableau 4. Distribution sociale des captives de la liste de Hotunskij

\begin{tabular}{|l|l|l|}
\hline & Nombre & Pourcentage \\
\hline Paysanne & 304 & $55 \%$ \\
\hline Inconnue & 136 & $25 \%$ \\
\hline Fille (femme) d'un cadet de province (syn bojarskij) & 69 & $12 \%$ \\
\hline Fille (femme) d'un cosaque & 19 & $3 \%$ \\
\hline Fille (femme) d'un mousquetaire & 16 & $3 \%$ \\
\hline Fille (femme) d'un prêtre & 6 & $1 \%$ \\
\hline Fille (femme) d'un taillable des villes (posadskij) & 3 & $0,5 \%$ \\
\hline Femme d'un commis (prikazčik) & 1 & $0,5 \%$ \\
\hline
\end{tabular}




\begin{tabular}{|l|l|l|}
\hline Ensemble & 554 & $100 \%$ \\
\hline
\end{tabular}
on remarque que les anciennes esclaves (holopy) sont tout à fait absentes. En même temps, les esclaves formaient une partie considérable de la population citadine et rurale en Moscovie, particulièrement dans les districts touchés par les incursions tatares. Selon Mihail N. Tihomirov, dans le district de Tula vers la fin $\mathrm{du} \mathrm{xvI}^{\mathrm{e}}$ siècle les esclaves formaient $30 \%$ de la population rurale, et les paysans (krest'jane) $45 \%{ }^{33}$ Comment les esclaves ont-elles pu être complètement absentes de la liste? En cherchant leurs traces, on tombe sur le groupe d'anciennes captives qui prétendaient ignorer leur identité sociale $(25 \%)$. On pourrait supposer que ce sont des esclaves, qui préférèrent une émancipation tacite en cachant leur ancienne identité sociale.

41 Cette hypothèse parait vraisemblable, mais elle se heurte à une contradiction. Les anciennes esclaves n'avaient aucune nécessité de cacher leur identité initiale, car la captivité et le rapatriement, selon la législation moscovite, émancipaient automatiquement de l'esclavage. Par contre, une norme identique n'existait pas pour les 
serfs, même si dans la pratique les deux cas étaient traités de la même manière, c'est-à-dire que les serfs étaient émancipés de leurs seigneurs pendant leur rapatriement. Par conséquent, il n'était pas avantageux pour les anciennes esclaves de se déclarer paysannes : elles risquaient d'échanger une émancipation immédiate garantie par la loi contre une possibilité incertaine de future émancipation. Malgré toutes ces raisons, on pourrait supposer que les esclaves tombaient aussi en captivité, et qu'en se rapatriant elles choisissaient soit de s'attribuer une nouvelle identité sociale, soit d'« oublier » leur ancienne identité sociale, en laissant les autorités les inscrire dans un des groupes sociaux de leur choix.

Ces deux exemples - les femmes de mousquetaires et de cosaques, qui sont sous-représentées, et les anciennes esclaves, qui sont absentes - suggèrent que le rapatriement présentait un certain choix de passerelles qui permettaient aux individus d'améliorer leur statut social ${ }^{34}$.

\section{Oublier et se souvenir}

En avançant la thèse sur la captivité et le rapatriement comme laboratoires de nouvelles identités sociales, nous n'avons réservé à nos personnages que deux options, soit indiquer leur vraie identité sociale, soit mentir, en la remplaçant par une autre, fictive, mais désirée. Pourtant, il existait une troisième option, celle de l'oubli, qu'on pourrait bien admettre chez les anciens captifs.

En caractérisant 121 personnages, c'est-à-dire, une sixième ou une septième partie de la liste de Hotunskij, les sous-secrétaires ont utilisé la remarque « ...ne se souvient pas (ne pomnit) ». Il faut rappeler que les informations biographiques sur les rescapés ne sont pas homogénéisées. On trouve parfois des omissions, qui ne sont pas introduites par cette formule («ne se souvient pas »). Il semble que les sous-secrétaires l'utilisaient dans le cas où le personnage ne pouvait ou ne voulait pas répondre à une question qui lui était posée. Deux explications pourraient être citées pour expliquer ce phénomène : la volonté de corriger ses origines locales et sociales, qui est constitutive pour la mobilité sociale, et l'amnésie. Pour vérifier ces explications, essayons d'étudier le groupe de ceux qui ont « oublié » leurs origines.

Tableau 5. La distribution de ceux qui ont « oublié leurs origines », par sexe et âge " $^{35}$

\begin{tabular}{|l|l|l|}
\hline & Anciens captifs & Anciennes captives \\
\hline Mineurs & & \\
\hline (« malyj », « mal'čik », & & \\
\hline « devočka » etc. & 21 & 16 \\
\hline Adultes & & \\
\hline (« žonka », « devka » etc. & 6 & 55 \\
\hline Non indiqué & 17 & 4 \\
\hline
\end{tabular}




\begin{tabular}{|l|l|l|}
\hline Total & 44 & 75 \\
\hline
\end{tabular}

Il faut dire que les «Ivan, qui ont oublié leurs familles (Ivany rodstva ne pomnjaščie) » ${ }^{36}$, oubliaient des choses différentes. Trente et un captifs et captives, c'est-à-dire une claire minorité, avaient oublié leur prénom. Dans ce cas, l'entrée individuelle dans la liste avait la forme suivante : «Un captif, qui s'est présenté comme Russe, ne sait pas son prénom, non plus que celui de son père, non plus que la ville ou le village d'où il est originaire; il ne peut pas se souvenir du temps qu'il a passé en captivité » (1.49, n²05). En revanche, la majorité des captifs (88) connaissaient leur prénom, parmi eux 35 savaient en plus leur patronyme ou leur nom, mais avait oublié leur identité locale et sociale : seuls 25 captifs et captives pouvaient indiquer la localité dont ils étaient originaires, et seuls 20 savaient le statut social de leurs parents.

L'interprétation de ces données est particulièrement difficile, car les historiens préfèrent toujours travailler avec des personnages qui parlent, et non pas avec ceux qui refusent de témoigner. Néanmoins, on pourrait d'abord avancer une hypothèse qui privilégie l'amnésie. Selon cette hypothèse, les enfants sont beaucoup moins capables de cacher leurs vraies identités ${ }^{37}$. Si on revient aux statistiques, il semble que cette hypothèse puisse s'appuyer sur la prédominance des captifs mineurs par rapport aux captifs adultes (21 contre 6). Bien sûr, parmi les rescapés et rescapées adultes on pourrait aussi trouver ceux qui furent capturés pendant l'enfance, ce qui aboutissait à l'effacement de toute information sociale sur l'individu, mais il est difficile de les identifier. Pour cela, nous aurions besoin dans chaque cas d'une indication précise de l'âge et de la date de capture.

En revanche, si nous privilégions l'option d'une tactique consciente, par laquelle on cachait son identité, on pourrait la soutenir dans le cadre de trois hypothèses. Premièrement, on pourrait supposer que l'« oubli » de son prénom est un moyen radical, par lequel on efface sa conversion à l'Islam et on revient à l'ancienne identité (dans ce cas, on cache son prénom). Deuxièmement, on pourrait supposer que les anciennes captives, qui pourraient avoir eu des expériences d'exploitation sexuelle en captivité, aient voulu cacher leur identité. Troisièmement, on pourrait supposer que les anciens captifs et captives choisissaient des identités sociales nouvelles plus élevées.

Dans le cadre de la deuxième hypothèse, les femmes devraient dominer dans le groupe de "ceux qui ont oublié leurs origines». De plus, on pourrait supposer chez elles une tendance à oublier leur identité locale, parce que pour celles qui se voyaient compromises par leurs expériences, il valait mieux de choisir un entourage social radicalement nouveau. En revenant aux statistiques, nous voyons une claire majorité féminine, ce qui correspond à la constellation générale de la liste de Hotunskij (66\% dans la liste de Hotunskij, $63 \%$ parmi ceux «qui ont oublié leurs origines»). Par contre, un détail pourrait favoriser cette hypothèse : parmi les captives «qui ont oublié leurs origines ", les adultes dominent clairement par rapport aux mineures (55 contre 16); autrement dit, la constellation est inversée en comparaison avec celle de captifs. Cette présence d'un groupe des captives adultes, qui préféraient de ne rien dire sur leurs origines, favorise clairement la deuxième hypothèse.

Dans le cadre de la troisième hypothèse, selon laquelle les captives agissaient de façon consciente et planifiée, cherchant une amélioration du statut social initial, ce soupçon semble assez fondé dans deux cas. Il s'agit de deux captives qui avaient oublié les noms et les lieux de résidence de leurs pères, mais qui se souvenaient que ces derniers étaient 
"cadets de province », c'est-à-dire porteurs du statut social le plus privilégié dans les districts voisins de la ligne. Ainsi, la captive Agafja Grigor'eva «se nomma fille de cadet de province, elle ne se souvient pas du nom ni de la ville de son père; elle fut capturée dans son enfance et resta en captivité vingt ans » (1. 14, $\mathrm{n}^{\circ}$ 53). La présentation de «la jeune fille captive Anna » est identique : « elle se nomma fille de cadet de province, ne se rappelle pas comment s'appelait son père, de quelle ville ou village il venait; elle fut capturée dans l'enfance et resta en captivité vingt ans " (1.23, $\left.\mathrm{n}^{\circ} 86\right)$. Dans d'autres circonstances, une falsification de statut social aurait été sévèrement punie, mais dans la situation particulière du rapatriement, où il était techniquement impossible de vérifier les récits des rescapés, les responsables moscovites faisaient preuve d'une tolérance exceptionnelle.

Dans quelle mesure les autorités pouvaient apprécier ces individus, qui, avec leurs oublis conscients ou inconscients, étaient comme les fiches vierges, qu'il fallait munir d'une nouvelle identité, classer ou reclasser ? De ce point de vue, les rapatriés présentaient un matériel presque idéal pour les premières tentatives de social engineering.

\section{La Moscovie comme une grande famille d'accueil?}

51 En notant minutieusement l'origine d'un individu ou d'un autre, les autorités moscovites semblent n'avoir jamais pensé à la possibilité de refuser l'entrée à quiconque. Par exemple à ceux qui, préférant les réalités moscovites aux réalités criméennes, voulaient tout simplement passer de l'autre côté de la frontière. On pourrait revenir sur le cas de Kutlukiz, cité au début de cet article, qui ne pouvait évoquer que vaguement ses origines moscovites. Pourtant les autorités restèrent fidèles à leur politique de porte ouverte.

De même pour les enfants, quelles que soient les différentes catégories. La paysanne Praskov'ja Rodionova (Kolomna) sortit de captivité avec son fils Kutlučjura, « qu'elle a conçu avec un Tatare ", n'oublie pas de remarquer le scribe ${ }^{38}$. Le prénom de Kutlučjura suggère qu'il a grandi dans un milieu musulman, cependant, nous ne savons pas s'il était circoncis, car ce dernier élément n'intéressait pas les autorités moscovites, qui n'ont jamais fait de remarques sur ce sujet. On pourrait supposer que le baptême avait été organisé pour Kutlučjura et les autres enfants de ce groupe. Les enfants capturés avec leur mère présentaient un cas d'intégration plus simple. Pour sept des onze enfants de ce groupe, nous connaissons leurs prénoms. Il s'agit de prénoms chrétiens : Lifan, Ostafej, Ignaška, Ivaška, Vas'ka, Ul'ka, Matrenka et Ogrofenka, ce qui suggère que ces enfants étaient déjà baptisés et ne furent pas convertis pendant la captivité. Sur les six enfants adoptifs, on n'en trouve que deux dont les prénoms sont évoqués, Griška et Nataška, mais dans ce cas il est possible que les autres aient vraiment oublié leur prénom. Le cas le plus difficile est présenté par le groupe d'enfants dont les origines ne sont pas évoquées. Les prénoms ici sont rares, et on suppose que les mères ne voulaient pas mentionner des prénoms turcs. Un grand champ de travail s'ouvrait ici devant les autorités moscovites.

Ce fait ne semble pas avoir changé la politique moscovite, bien que la situation familiale des individus qui venaient de toucher le sol moscovite ait pu poser plusieurs problèmes. La prédominance féminine avec un grand pourcentage de mères célibataires signifiait que seule une minorité des rapatriés pouvait se transformer en Moscovites taillables. La majorité des rapatriées devait dépendre de l'aide de l'État. Cette aide pouvait venir du Trésor, sous forme d'allocation ponctuelle pour le rapatriement (za vyhod), ou être déléguée aux communautés locales. 

mousquetaires (strel'cy), les sous-secrétaires (pod'jačie), etc. Cette mesure est très intéressante. Cela signifie que les autorités moscovites ne craignaient pas de concentrer dans la capitale des rapatriées en situation précaire. Pourtant, ces rapatriées et leurs récits sur le passé pouvaient alimenter un discours très hostile à l'égard des Tatares de Crimée. Peut-être considérerait-on les choses de manière trop moderne en voyant dans cette action des autorités moscovites une tentative de gagner le soutien populaire à leur politique offensive sur la frontière du Sud. Toutefois, même si les autorités moscovites ne prévoyaient aucune propagande visant leurs propres populations, une ancienne captive au seuil de la maison moscovite pouvait représenter une " gazette vivante $»^{39}$.

ent sur les aspects démographiques des deux approches opposées, celle de Baghčesaray et celle de Moscou, on pourrait les comparer avec les théories sur les sources de richesse des nations du XVII et du XVIII ${ }^{\mathrm{e}}$ siècle. De ce point de vue, la politique du Khanat de Crimée représentait un mercantilisme rude, mené jusqu'au bout, quand on vendait systématiquement des esclaves pour obtenir de l'argent (l'ouverture pour le rachat s'inscrivait dans ce modèle). En revanche, la vision moscovite semble comparable aux théories du peuplement, élaborées beaucoup plus tard. Inébranlables dans leur politique de «porte ouverte ", les autorités moscovites ne recevaient pas seulement leurs anciens sujets, y compris des individus qui avaient besoin d'aide à l'intégration, mais aussi ceux qui voulaient tout simplement traverser la frontière et affichaient des identités pour le moins douteuses ${ }^{40}$. Cependant on ne doit pas être déterministe et attribuer le développement ultérieur, y compris les résultats impressionnants de l'expansion russe au $\mathrm{XVIII}^{\mathrm{e}}$ siècle, à l'échec de la première politique et aux succès de la seconde.

\section{NOTES}

1. RGADA (Rossijskij gosudarstvennyj arhiv drevnih aktov - Archives d'État de Russie des actes anciens), f. 123 (Snošenija s Krymom [Les relations avec la Crimée]), op. 1, 1649, n 7, 1.1-313.

2. «Polonjanka žonka Ul'jana Kurčenina syna bojarskogo Trofimova doč' Malyhina, v polonu byla 25 let. U nej dve devočki sirotki prijmyši, odna Nataška Ivanova doč' Kobylinoj derevni, kotorogo goroda i uezdu, togo ne pomnit, let v pjat', a drugaja devočka goda v dva, imeni sebe i otca ne znaet, vzjata devočka sej ž vesny » $\left(1.19, \mathrm{n}^{\circ} 70\right)$.

3. «Polonjanka devka, sebe imeni i otca ne pomnit, vzjata v polon nevelika, sego leta ( $1.89, \mathrm{n}$ $\circ 285)$.

4. «Polonjanka, devka, 15 let, skazalas', chto ona rodilas' ot ruski(h), Kutlukizom zovut» (1. 139). La question cruciale est celle de la véracité des récits des anciennes captives : le chercheur ne peut vérifier les données de la liste par des sources indépendantes. Même dans un rare cas où l'on trouve des suppliques de captifs figurant sur la liste, on pourrait supposer que ces dernières répètent les mêmes « histoires » autobiographiques que celles qui se reflètent dans les courtes notices biographiques de la liste.

5. V.V. Radlov Opyt slovarja tjurkskih narečij [Essai de dictionnaire des dialectes turcs], t. 2, SPb., 1899, p. 818-819, 996-997. Sur les doubles identités religieuses et le rôle de la conversion dans l'intégration des Tatares de Crimée en Moscovie, voir : A. Lavrov, « Le tsar, le poète et le converti. 
Le rôle de la conversion dans les relations entre le Khanat de Crimée et la Moscovie au XVII siècle ", Les mutations religieuses en Russie : conversions et sécularisation, éd. par Françoise Lesourd (Slavica occitania, 42, 2015), Toulouse, 2015, p. 43-59.

6. Il est très intéressant de comparer ces données à celles de Vjačeslav D. Žukov qui a étudié les listes des captifs rachetés pendant le rachat "centralisé ", qui était organisé une fois par an à Valujki et largement financé par le Trésor. L'historien a choisi les années 1651 et 1655, ce qui est particulièrement intéressant, parce que ses données sont presque contemporaines des nôtres (1649). Ici la majorité absolue était masculine (145 personnes), tandis que les captives ne formaient qu'un groupe minuscule (25 personnes). Žukov trouve que «la majorité absolue des femmes capturées fut transférée aux marchés des esclaves de Méditerranée, sans pouvoir revenir dans leur patrie », (V.D. Žukov, " "Krymskie polonjaniki” i ih vykup v 50-h gg. XVII v. : k istorii kolonizacii južnoj okrainy Moskovskogo gosudarstva [Les "polonjaniki (anciens captifs)" de Crimée et leur rachat dans les années 1650 : contribution à l'histoire de la colonisation des confins méridionaux de l'État moscovite]», Vestnik Rossijskogo universiteta družby narodov. Serija Istorija Rossii, 2012, n 4, p. 31-43, ici p. 40). La présence de nombreuses captives dans la liste de Hotunskij montre que ce n'était pas le cas et que des centaines de captives d'origine moscovite restaient en Crimée. Je crois que la cause de cette différence entre les deux listes - la majorité féminine dans la liste de Hotunskij et la majorité masculine dans les listes de Valujki résidait dans le caractère élitaire du rachat «centralisé ». Les autorités moscovites préféraient racheter les gens de service, y compris ceux qui tombaient en captivité pendant leur mission ou sur le champ de bataille, ce qui était compréhensible, mais les femmes devenaient les principales perdantes d'une telle politique.

7. Nancy Shields Kollmann, «Women's Honor in Early Modern Russia», in Barbara Evans Clements, Barbara Alpern Engel, Christine D.Worobec, eds., Russia's Women: Accomodation, Resistance, Transformation, Berkeley, 1991, p. 60-73.

8. Nada Boškovska, Die russische Frau im 17. Jahrhundert, Köln, 1998. Voir mon compte-rendu : Otečestvennaja istorija, 1999, n 4, p. 177-179.

9. La seule décision très importante, portant sur les captives, à savoir la permission d'échanger une captive moscovite contre un captif tatare, fut prise par le tsar Fedor Alekseevič. Le niveau, exceptionnel, auquel fut prise cette décision, ainsi que son caractère tardif, soulignent le statut subordonné des femmes dans la politique du rachat (voir: A. Lavrov, «Captivity, Slavery and Gender: Muscovite Female Captives in the Crimean Khanate and in the Ottoman Empire ", in Christoph Witzenrath, ed., Eurasian Slavery, Ransom and Abolition in World History, 1200-1860, Farnham, 2015, p. 309-319).

10. V.P. Zagorovskij, Belgorodskaja čerta [La ligne Belgorod], Voronež, 1969, p. 72. Dans cette étude qui reste une référence, V.P. Zagorovskij réussit à élaborer une vision de la société moscovite qui différait clairement de la «ligne générale» de l'historiographie soviétique. L'historien montra comment une large "ingénierie sociale» servait à la colonisation de nouveaux territoires et à leur défense, et non aux intérêts d'une "classe » particulière. Se cachant habilement derrière un sujet local, Zagorovskij réussit à rendre sa vision publique, sans provoquer d'orage de la part des autorités.

11. Les villes de Valujki et Voronež étaient aussi classées, pendant les années 1620, comme «villes de la steppe ». Je les ajoute au premier groupe, parce que vers 1649 ces villes faisaient partie de la ligne Belgorod en pleine construction (Zagorovskij, Belgorodskaja @erta, p. 21).

12. Voir: A. Jakovlev, Zasečnaja čerta Moskovskago gosudarstva v XVII veke : Očerk iz istorii oborony južnoj okrainy Moskovskago gosudarstva [La ligne d'abattis de l'État moscovite au XVII ${ }^{\mathrm{e}}$ siècle : de l'histoire de la défense des confins méridionaux de l'État russe], M., 1916 (avec une carte détaillée). 
13. M.N. Tixomirov, Rossija v XVI stoletii [La Russie au XVI ${ }^{\mathrm{e}}$ siècle], M., 1962, p. 376. Chez Tihomirov, Odoev, Kaluga, Kozel'sk sont aussi classées dans ce groupe, tandis que dans mon classement elles sont distribuées entre le troisième et le quatrième groupe.

14. Tihomirov, Rossija v XVI stoletii, p. 371.

15. Zagorovskij, Belgorodskaja čerta, p. 33, 49.

16. Sur la construction et le développement de cette bourgade, voir la monographie de Davies.

B.I. Davies, State, Power and Community in Early Modern Russia : The Case of Kozlov, 1635-1649, Houndmills, 2004.

17. Tihomirov, Rossija v XVI stoletii, p. 417.

18. A.A. Novosel'skij, Bor'ba Moskovskogo gosudarstva s tatarami v pervoj polovine XVII v. [La lutte de l'État moscovite contre les Tatares dans la première moitié du XVII ${ }^{\mathrm{e}}$ siècle], M.-L., 1948, p. 10.

19. Zagorovskij, Belgorodskaja čerta, p. 54 .

20. G.A. Sanin, «Južnaja granica Rossii vo 2-j polovine XVI - 1-j polovine XVIII vv. [La frontière sud de la Russie, seconde moitié du XVI ${ }^{\mathrm{e}}$ - première moitié du XVIII ${ }^{\mathrm{e}}$ siècle] ", Russian History, vol. 19, n 1-2, 1992 (Frontier in Russian History), p. 433-457, ici p. 445-450.

21. Il est intéressant de noter que la durée de la captivité des rachetés, qui furent rançonnés à Valujki pendant les années 1651 et 1655, était moins grande. Selon Vjačeslav D. Žukov, seuls $29 \%$ d'entre eux passèrent en captivité vingt ans et plus. La majorité de ces captifs ne furent pas captifs plus de dix ans, et un quart d'eux y passa moins de cinq ans. «On ne rencontre que rarement des individus qui passèrent plusieurs décennies loin de leur patrie ", écrit l'historien (Žukov, «"Krymskie polonjaniki” i ih vykup... », p. 39). Toutes ces données, largement crédibles, ne prouvent qu'une chose: les gens de service, qui étaient l'objet principal du rachat " centralisé », étaient tellement privilégiés qu'ils passaient moins de temps en captivité que les captifs « ordinaires».

22. Bien sûr, les indications données par les captives sont parfois imprécises. Cela concerne surtout 156 captives, qui ont indiqué qu'elles passèrent «vingt ans » en captivité. Dans ce cas, « vingt ans » signifie tout simplement « depuis longtemps ».

23. " welche aber bei jnen verkaufft oder behalten werden / die dienen sich mit Sechs jaren frey / aber aus dem Landt thuern sy nit ziehen/ dienen und wie sich ein yeglicher zu seiner weittern narung schickhen khan. " Sigismund von Herberstein, Rerum Moscoviticarum Commentarii, synoptische Edition der lateinischen und der deutschen Fassung letzter Hand, Basel 1556 und Wien 1557. Unter der Leitung von Frank Kämpfer erstellt von Eva Maurer und Andreas Fülberth. Redigiert und herausgegeben von Hermann Beyer-Thoma, München 2007, p. 308 (qui autem venduntur, perpetuo sexennio servitutem servire coguntur: quo exacto, liberi quidem fiunt, provincia tamen decedere non audent).

24. Mihalon Litvin, O nravah tatar, litovcev i moskvitjan [Michalonis Lituani (Michel le Lituanien), De moribus Tartarorum, Lituanorum et Moschorum fragmina X... Basileae, 1615, p. 26], M., 1994, p. 89. Il semble que l'information de Michalon Lituanus dépende de celle de Herberstein. Mikhail Kizilov qui ne cite que le témoignage de Michalon Lituanus, ne remarque pas cette dépendance (M. Kizilov, « Slave Trade in the Early Modern Crimea from the Perspective of Christian, Muslim, and Jewish Sources », Journal of Early Modern History, 11 (1-2), 2007, p. 1-31, ici p. 14).

25. Les données sur l'âge des enfants manquent parfois, mais on peut calculer l'âge moyen : 5 ans et quatre mois.

26. « Polonenik Ivaško Terent'ev karačevec, krest'janskoj syn, u nevo žena Orina Ivanova doč', karačevka, vzjaty v polon tomu 20 let » (l. 24).

27. «Polonenik Stepan Dmitreev krest'janskoj syn kotorogo goroda i derevni, togo ne upomnit, vzjat v polon tomu 30 let, u nego žena polonjanka Matrenka Grigor'eva krest'janskaja doč' Pereslavlja Rezanskogo iz derevni iz Lukinoj, vzjata v polon tomu 20 let. U nej syn 5 let, da na 
rukax dočka devočka nevelika, 2 godov... Polonenik Ivan Stepanov Borisova goroda krest'janskoj syn byl v polonu 22 goda. U nego žena evo Fedos'ja Romanova Kolužskogo uezdu i prižil s neju dva deti devka let 10, drugaja 4 let » (1. 44, n 176-177, 179-180).

28. « Polonenik Ivaško Vasil'ev belevskogo goroda krest'janskoj syn, byl v polonu 25 let. U nego žena Marinka Jur'eva doč' da syn 7 let, prižila v nagaeh » (l. 32 ( $\left.n^{\circ} 126-127\right)$.

29. Bien sûr, le devşirme, le système d'enlèvement des garçons chrétiens, séparés de leurs familles, convertis et éduqués comme janissaires, semble n'avoir jamais fonctionné au Nord de la Mer Noire. De plus le devşirme fut aboli dans l'Empire ottoman vingt ans avant la composition de la liste de Hotunskij, ce qui contribua à la « nationalisation » du corps des janissaires.

30. Par contre, une explication alternative pourrait aussi être admise : tandis que la population rurale était capturée pendant les incursions tatares, les résidentes des villes et des bourgades prises d'assaut, pourraient avoir été massacrées pendant le sac, ce qui expliquerait leur pourcentage minuscule parmi les captives.

31. Voir les données pour les «villes dans la steppe » en 1626 (Zagorovskij, Belgorodskaja Certa, p. 27). Il est particulièrement intéressant que les gens de service sur contrat (po priboru) forment la majorité des rachetés pendant le rachat centralisé à Valujki (52,3\%) (Žukov, " "Krymskie polonyniki" i ix vykup », p. 36). Ici on voit clairement la différence entre les deux sources, qui résulte de leur origine. Pendant le rachat «centralisé », les rançons furent payées par le Trésor, ce qui transformait toute tentative individuelle de s'attribuer un statut social élevé en une manipulation malhonnête. Lors d'actions semblables à celle de Hotunskij, quand les affranchis par leurs propriétaires et rachetés par le Trésor ou par leurs familles sortaient ensemble, les règles étaient moins strictes.

32. Zagorovskij, Belgorodskaja čerta, p. 28-29.

33. Tihomirov, Rossija v XVI stoletii, p. 381.

34. La mobilité sociale en Moscovie n'est pas devenue jusqu'à présent un objet privilégié de la recherche. On ne pourrait citer que les travaux de Viktor Borisov sur la mobilité sociale dans le milieu paysan et sur les "gens de passage » (guljaščie ljudi), ainsi que l'article de Tat'jana Lapteva sur le recrutement de représentants de différents groupes sociaux comme « cadets de province » (V.E. Borisov, Irbitskaja sloboda v XVII veke : social'naja struktura i povsednevnye otnošenija v lokal'nom soobščestve [Le bourg d'Irbit au XVII ${ }^{\mathrm{e}}$ siècle : structure sociale et relations au quotidien au sein de la société locale], Avtoreferat... kandidata istoriceskix nauk, M., 2010 ; T.A. Lapteva, « Poverstanie v deti bojarskie predstavitelej drugix soslovij : K voprosu o rasširenii social'noj bazy dvorjanskogo soslovija v XVII veke [Le recrutement de représentants de différents groupes sociaux comme cadets de province ou de l'élargissement de la base sociale de la classe des aristrocrates au XVII ${ }^{\mathrm{e}}$ siècle]», Otečestvennaja istorija, 2003, $\mathrm{n}^{\circ} 5, \mathrm{p}$. 81-96).

35. Dans ce tableau sont absents deux enfants, dont le sexe n'est pas précisé dans la liste : « dvoe robjat malen'kie, odin v sem', a drugoj let četyrex, imen sebe ne pomnjat » (l. 29, $\left.\mathrm{n}^{\circ} 108-109\right)$.

36. Je n'ai jamais rencontré une tentative de commentaire historique à cette expression idiomatique. Tenant compte de l'absence d'explications alternatives et du parallélisme étonnant avec certaines entrées à la liste de Hotunskij, je pense qu'il ne s'agit tout simplement pas d'individus qui ne peuvent rien dire sur leurs origines, mais plutôt d'un phénomène d'oubli, causé par leur séjour en captivité.

37. Ici on doit être prudent, car on ne pourrait déclarer chaque enfant qui ne pourrait rien dire de soi comme un cas d'amnésie. On pourrait attendre d'une fillette de trois ans de connaître son prénom et ceux de ses parents, ainsi, son incapacité de les nommer pourrait être expliquée par le traumatisme de la capture (1. 144, $\mathrm{n}^{\circ}$ 606). Par contre, on ne pourrait attendre la même chose des deux enfants de deux ans, qui sont aussi inscrits dans la liste. Dans ce cas il est impossible de parler d'amnésie.

38. «Polonjanka žonka Praskov'ja Rodivonova, s Kolomny krest'janskaja doč', u nej syn Kutlučjura, prižila s tatarinom » $\left(1.55, n^{\circ} 234\right)$. 
39. En un certain sens, cette obligation inhabituelle de nourrir et de loger des rapatriés chez soi rappelle la pratique d'un impôt spécial, qui frappait la population taillable : la taxe destinée au rachat des prisonniers (polonjaničnye den'gi). Cette taxe, dont les revenus n'étaient pas seulement utilisés pour le rachat, mais aussi pour d'autres dépenses, pourrait aussi être vue comme un moyen de rappeler aux Moscovites leur responsabilité collective concernant le sort des captifs se trouvant au Khanat de Crimée et dans l'Empire ottoman.

40. Sur l'intégration de rapatriés, voir : A. Lavrov, « Polonjaniki kak social'naja gruppa : pravovoj status i reintegracija byvših voennoplennyh v Moskovskom gosudarstve [Les anciens captifs (polonjaniki), groupe social en Moscovie : statut juridique et réintégration factice ", Cahiers du Monde russe, 51 (2-3), 2010, p. 241-258.

\section{RÉSUMÉS}

L'article est consacré à une nouvelle source concernant les relations entre la Russie et la Crimée : une liste de captifs russes rapatriés de Crimée par les soins de l'envoyé du tsar Timofej Hotunskij. Elle se caractérise par une nette prédominance des femmes sur les hommes (554 contre 169). Ces captives, qui n'avaient pas pris part à des combats, permettent de reconstituer le tableau des pertes démographiques subies par la population civile et de localiser les régions qui avaient le plus souffert des incursions tatares. Cette recherche permet de comprendre la logique des autorités moscovites et criméennes. Pour ces dernières, la prise de captifs et l'esclavage étaient une source de revenu, de sorte qu'elles ne s'opposaient pas, dans le principe, à l'affranchissement d'esclaves déjà âgés qui devenaient une charge improductive pour leurs propriétaires. Au lieu que les autorités moscovites étaient prêtes à reprendre tous leurs anciens sujets, même s'il leur en coûtait des dépenses supplémentaires. Il en était ainsi lorsqu'il s'agissait d'hommes âgés et de femmes qui revenaient avec des enfants nés en captivité. Il y avait donc là deux politiques démographiques opposées : tandis que les Criméens concoururent à expulser du Khanat des groupes entiers de population, les Moscovites préféraient pratiquer la politique des «portes ouvertes ".

The article analyzes a new source on the relations between Muscovy and the Khanate of Crimea a list of names of Muscovite captives who returned home with the help of the Muscovite envoy Timofei Khotunskii. The particularity of this list is the predominance of female captives (554 female versus 169 male captives). The female captives, as classical non-combatants, give a representative picture of the losses of the Muscovite civil population from incursions by the Tatars of Crimea and permit to locate the regions that were the most damaged by these incursions. The study helps to understand the logic of the Muscovite and Tatar authorities. The Crimean policy makers viewed captivity and slavery as a profitable matter and were not opposed to releasing old captives, who were useless to their owners. The Muscovite policy makers present the opposite logic: they proceeded to the repatriation of any Muscovite captives, including the elderly and young mothers with children, even though this population group needed social assistance from the state. The demographic policies of these early modern states thus seem to be opposite to one another - the Crimean policy makers systematically proceeded to expulse people, while the Muscovite policy makers practiced an "open-door" policy. 


\section{AUTEUR}

\section{ALEKSANDR LAVROV}

Université Paris-Sorbonne Département d'études slaves, allavrov@yahoo.com 